\title{
Polysaccharides with Antioxidative and Antiaging Activities from Enzymatic-Extractable Mycelium by Agrocybe aegerita (Brig.) Sing
}

\author{
Huijuan Jing, ${ }^{1}$ Qing Zhang, ${ }^{2}$ Min Liu, ${ }^{1}$ Jianjun Zhang $\left(D,{ }^{1}\right.$ Chen Zhang, \\ Shangshang Li, ${ }^{1}$ Zhenzhen Ren, ${ }^{1}$ Zheng Gao, ${ }^{1}$ Xingtian Liu $\mathbb{D},{ }^{3}$ and Le Jia $\mathbb{D}^{1}$ \\ ${ }^{1}$ College of Life Science, Shandong Agricultural University, Tai'an 271018, China \\ ${ }^{2}$ College of P.E \& Art, Shandong Agricultural University, Tai'an 271018, China \\ ${ }^{3}$ Taian City Central Hospital, Tai'an 271018, China \\ Correspondence should be addressed to Xingtian Liu; liuxt_123@163.com and Le Jia; jiale0525@163.com
}

Received 10 February 2018; Revised 25 May 2018; Accepted 18 July 2018; Published 4 December 2018

Academic Editor: Svein Haavik

Copyright (C) 2018 Huijuan Jing et al. This is an open access article distributed under the Creative Commons Attribution License, which permits unrestricted use, distribution, and reproduction in any medium, provided the original work is properly cited.

This study aimed to investigate the antioxidant, antiaging, and organ protective effects of the water-extractable mycelium polysaccharides (MPS) and enzymic-extractable mycelium polysaccharides (En-MPS) by Agrocybe aegerita (Brig.) Sing in Dgalactose-induced (D-gal-induced) aging mice. In in vitro assays, the En-MPS demonstrated stronger antioxidant activities in dosedependent manners. The mice experiments revealed that both En-MPS and MPS had potential effects on antioxidation, antiaging, and organ protection mainly by improving the antioxidant enzyme activities, decreasing the lipid peroxidation, and remitting the lipid metabolism. Furthermore, chemical composition and monosaccharide composition of polysaccharides were also measured, and the results indicated that differences in biological activity of MPS and En-MPS samples showed a significant correlation to their purity. The findings demonstrated that the polysaccharides by A. aegerita (Brig.) Sing could be exploited as natural and functional foods for the prevention and alleviation of aging and its complications.

\section{Introduction}

Aging is an inevitable natural phenomenon that is characterized by an accumulation response for dysregulation of immunity, decline of functions, atrophy of tissues, wrinkling of skin, etc. [1-3]. Previous studies have demonstrated that aging is involved in many diseases, including cancers, neurodegenerative diseases, and cardiovascular diseases [4]. Growing evidence from scientific studies has suggested that oxidative damage from reactive oxygen species (ROS), which are generated by metabolism and have been shown to cause molecular damage relatively indiscriminately to proteins, lipids, and nucleic acids, can cause cumulative cellular and organic damage and play crucial roles in aging-induced skeletal muscle decline, accelerating aging progress $[1,2,5-$ 7].

Antioxidants are commonly used as antiaging agents, contributing to their potential effects on scavenging reactive radicals [8]. However, synthetic antioxidants are restrictively used due to their side effects on health during long-term administration [9]. Hence, it is necessary and important to explore natural and nontoxic antioxidants. Mushrooms are considered perfect foods not only due to their unique taste and high nutrition but also due to their low-calorie, lowcholesterol, low-sodium, and high-protein properties [10]. It has been demonstrated that polysaccharides extracted from mushrooms play vital roles in maintaining healthpromoting activities, such as antiobesity [11], antiinflammatory [12], antioxidative [3], and hepatoprotective effects [13]. Hence, increasing studies have demonstrated that mushroom polysaccharides have emerged as good alternatives for the delaying aging progress $[8,14]$.

Agrocybe aegerita (Brig.) Sing, belonging to Basidiomycete and called "Yangshugu" in China, is appreciated as an edible and medicinal mushroom among Chinese folk people, which may be due to its traditional antimicrobial effects and 
improvement of nephritis and edema $[15,16]$. Previous studies have indicated that polysaccharides are the most abundant substances in mushrooms and have become an important constituent in the research and development of natural drugs and healthcare products [17]. However, few reports about the antioxidant and antiaging activities of mycelium polysaccharide from $A$. aegerita (Brig.) Sing have been published. Meanwhile, enzymatic-extractable-extraction technology is widely used in polysaccharide extraction due to its high extraction yield and reproducibility [18]. Taken together, it is quite meaningful to explore natural agents with potential antioxidant and antiaging effects for preventing and postponing aging and its complications.

The aim of the present work was to evaluate the antioxidative and antiaging effects of the mycelium polysaccharides (MPS) and its enzymatic-hydrolysate of enzymaticMPS (En-MPS) from A. aegerita (Brig.) Sing against Dgalactose-induced (D-gal-induced) aging, aiming to obtain a better understanding of their possible antiaging mechanisms. Additionally, the monosaccharide compositions of En-MPS and MPS were also analyzed.

\section{Materials and Methods}

2.1. Materials and Reagents. The strain of A. aegerita (Brig.) Sing used in the present work was supplied by our laboratory, and liquid fermentation technology was applied on a cylinder (Luoyang, China) with a medium of glucose $(20 \mathrm{~g} / \mathrm{L})$, potato $(200 \mathrm{~g} / \mathrm{L}), \mathrm{KH}_{2} \mathrm{PO}_{4}(1.5 \mathrm{~g} / \mathrm{L})$, and $\mathrm{MgSO}_{4}$ $(1 \mathrm{~g} / \mathrm{L})$. Standard monosaccharide samples were purchased from Sigma Chemicals Company (St. Louis, USA). The diagnostic kits for assaying the activities of superoxide dismutase (SOD), catalase (CAT), and total antioxidant capacity ( $\mathrm{T}$ AOC), as well as the contents of malondialdehyde (MDA) and hydroxyproline (Hyp), were purchased from Nanjing Jiancheng Bioengineering Institute (Nanjing, China). All other reagents and chemicals were of analytical grade and purchased from local chemical suppliers.

2.2. Preparation of En-MPS and MPS. The En-MPS of $A$. aegerita (Brig.) Sing was prepared according to the method of Lin et al. [19] with a slight modification. The dried mycelium was crushed into powder using a disintegrator (Shanghai, China) and sieved through a No. 80 mesh. The sifted powder was processed by enzymatic extraction with snailase solution ( $4 \%$, dissolved in acetate buffer, w/v) at $37^{\circ} \mathrm{C}$ for $6 \mathrm{~h}$ to obtain En-MPS, while using hot water extraction to gain MPS. After centrifugation $(3,600 \mathrm{r} / \mathrm{min}, 15 \mathrm{~min})$, the supernatants were precipitated with three volumes of ethanol (95\%, v/v) overnight at $4^{\circ} \mathrm{C}$. The carbohydrate contents were determined by the phenol sulfuric acid colorimetric method [20]. After deproteinization by Sevag [21] and dialysis against deionized water, both En-MPS and MPS were lyophilized for further experiments.

2.3. Antioxidant Activity In Vitro. The scavenging activity on DPPH was determined according to a previous method [22]. The reaction mixture contained DPPH ethanolic solution
$(2 \mathrm{~mL}, 0.1 \mathrm{mmol} / \mathrm{L})$ and polysaccharide solutions $(2 \mathrm{~mL}, 0$ $4,500 \mu \mathrm{g} / \mathrm{mL})$. After the 30-minute dark incubation, the absorbance at $517 \mathrm{~nm}$ was measured. The DPPH scavenging rates were calculated using the following equation:

$$
\text { Scavenging rates }(\%)=\left(1-\frac{\left(\mathrm{A}_{1}-\mathrm{A}_{2}\right)}{\mathrm{A}_{3}}\right) \times 100
$$

where $A_{1}$ was the absorbance of $2 \mathrm{~mL}$ polysaccharide solution and $2 \mathrm{~mL} \mathrm{DPPH}$ ethanolic solution, $\mathrm{A}_{2}$ was the absorbance of $2 \mathrm{~mL}$ polysaccharide solution and $2 \mathrm{~mL}$ absolute ethanol, and $\mathrm{A}_{3}$ was the absorbance of the $2 \mathrm{~mL}$ distilled water and $2 \mathrm{~mL}$ DPPH ethanolic solution.

The reducing power was measured according to the method of Oyaizu (1986) with minor modifications [23]. Polysaccharide solution $(1 \mathrm{~mL}, 0-4,500 \mu \mathrm{g} / \mathrm{mL})$, phosphatebuffered saline $(2.5 \mathrm{~mL}, 0.2 \mathrm{~mol} / \mathrm{L}, \mathrm{pH} 6.6)$, and potassium ferricyanide solution $(1 \mathrm{~mL}, 1 \%, \mathrm{w} / \mathrm{v})$ were put into the test tube sequentially. Trichloroacetic acid $(2 \mathrm{~mL}, 10 \%, \mathrm{w} / \mathrm{v})$ and ferric trichloride $(1.2 \mathrm{~mL}, 0.01 \mathrm{~g} / \mathrm{L}, \mathrm{w} / \mathrm{v})$ were added to the mixture after a 20 -minute incubation $\left(50^{\circ} \mathrm{C}\right)$. The absorbance of the mixture was analyzed at $700 \mathrm{~nm}$.

The scavenging activity on hydroxyl radicals was measured according to the previous method with a few modifications [24]. The reaction mixture of ferrous sulfate $(1 \mathrm{~mL}$, $9 \mathrm{mmol} / \mathrm{L})$, salicylic acid $(1 \mathrm{~mL}, 9 \mathrm{mmol} / \mathrm{L}$, dissolved in ethanol), tested samples ( $1 \mathrm{~mL}, 0-4,500 \mu \mathrm{g} / \mathrm{mL})$, and hydrogen peroxide $(1 \mathrm{~mL}, 8.8 \mathrm{mmol} / \mathrm{L}, \mathrm{w} / \mathrm{v})$ was incubated at $37^{\circ} \mathrm{C}$ for $30 \mathrm{~min}$, and the absorbance was measured at $510 \mathrm{~nm}$ using distilled water as a blank. The hydroxyl radical-scavenging rates were expressed as

$$
\text { Scavenging rates }(\%)=\frac{\left(A_{0}-A_{1}\right)}{A_{0}} \times 100
$$

where $A_{1}$ was the absorbance of the sample and $A_{0}$ was the absorbance of the blank.

2.4. Animal Experiments. Kunming strain male mice (weighing $20 \pm 2 \mathrm{~g}$ ) were purchased from the Taibang Biological Products Limited company (Tai'an, China). The animals were housed in polypropylene cages bedding in an airconditioned room with a temperature of $22 \pm 2^{\circ} \mathrm{C}$, humidity of $55 \pm 5 \%$, and a light/dark $(12 \mathrm{~h} / 12 \mathrm{~h})$ cycle, during which time all mice received rodent laboratory chow and water. All experiments were performed in accordance with the Regulations of Experimental Animal Administration issued by the State Committee of Science and Technology of the People's Republic of China.

After a 7-day acclimatization, all the mice were randomly divided into seven groups (five in each group), namely, the normal control group (NC group), model control group (MC group), positive control group (PC group), and four dosage groups of En-MPS and MPS. All aging mice except that in the NC group were successfully induced by intraperitoneal injection with D-gal at a dose of $200 \mathrm{mg} / \mathrm{kg}$, using isometric volumes of normal saline as blank in the NC groups. During the gavage procedure, the mice in the $\mathrm{PC}$ group were intragastrically administered vitamin $\mathrm{C}$ at a dose of $100 \mathrm{mg} / \mathrm{kg}$, 
and the mice in the dosage groups were administered polysaccharides at doses of 200 and $600 \mathrm{mg} / \mathrm{kg}$, respectively, using normal saline as a blank in the NC and MC groups. The D-gal injection and polysaccharide administration were processed alternatively, and the entire experiment lasted for 30 successive days.

At the end of the experiment, all mice were sacrificed under anaesthesia overnight fasting. Blood was collected to obtain serum by centrifugation $(12,000 \mathrm{r} / \mathrm{min}, 10 \mathrm{~min})$. The levels of total cholesterol (TC), triacylglycerol (TG), albumin (ALB), high-density lipoprotein cholesterol (HDLC), and low-density lipoprotein cholesterol (LDL-C), as well as the activities of alanine aminotransferase (ALT), aspartate aminotransferase (AST), and alkaline phosphatase (ALP) in serum, were measured using an automatic biochemical analyzer (ACE, USA).

The brain, liver, and kidney were immediately removed, washed with ice-cold normal saline, and homogenized (1:9, $\mathrm{g} / \mathrm{mL})$ in phosphate buffer solutions $(0.2 \mathrm{~mol} / \mathrm{L}, \mathrm{pH} 7.4)$. After centrifugation $\left(3,000 \mathrm{r} / \mathrm{min}, 4^{\circ} \mathrm{C}, 20 \mathrm{~min}\right)$, the supernatants were collected for further analysis. The SOD activities, CAT activities, T-AOC activities, and MDA levels were assayed according to the instructions of commercially available diagnostic kits. The dorsal skin $(0.5 \mathrm{~g})$ without hair was immediately collected and hydrolysed with $\mathrm{HCl}(6 \mathrm{~mol} / \mathrm{L}$, $\mathrm{w} / \mathrm{v}, 110^{\circ} \mathrm{C}$ ) for $6-12 \mathrm{~h}$, and the supernatants were collected for Hyp analysis according to the manufacturer's instructions.

For histological examinations, the brain, liver, and kidney were fixed in $10 \%$ formalin ( $\mathrm{pH} 7.4$ ), embedded in paraffin, sectioned by a slicer ( $5 \mu \mathrm{m}$ thickness), and stained with hematoxylin-eosin. The observations were processed under the microscope at $400 \times$ magnification for evaluations of morphological and pathological changes.

2.5. Acute Toxicity Study. The acute toxicity test in mice was performed according to the method of Chao et al. [25] with some modifications. Thirty-five Kunming strain mice were divided into one control group and six dose groups (five in each group), which received MPS and En-MPS at increasing dosages of 600,900 , and $1,500 \mathrm{mg} / \mathrm{kg}$, respectively. The experimental mice were allowed for food ad libitum and continuously observed for any behavioural changes, toxic symptoms, and mortality during the whole feeding period.

2.6. Chemical Composition Analysis. The total polysaccharide was determined by the phenol sulfuric acid colorimetric method [20]. Protein was determined according to Bradford method, using bovine serum as the standard [26]. The content of total phenolic was estimated using the Folin-Ciocalteu method [27].

2.7. Monosaccharide Composition Analysis. The monosaccharide compositions were determined by gas chromatography (GC) (GC-2010, Shimadzu, Japan) according to the previous method [28]. Sugar identifications were processed by comparisons with standard sugars in our previous study, including xylose (Xyl), arabinose (Ara), glucose (Glc), rhamnose (Rha), galactose (Gal), ribose (Rib), fucose (Fuc), and mannose (Man) [29]. The relative molar proportions were calculated by the area normalization methods.

2.8. Statistical Analysis. All the data were expressed as the mean \pm SD (standard deviation). Differences between experimental groups were assessed by one-way analysis (ANOVA, SPSS 16.0). $\mathrm{p}<0.05$ was accepted as statistically significant.

\section{Results}

3.1. In Vitro Antioxidant Activities Assays. The in vitro antioxidant activities of En-MPS and MPS were determined by the scavenging capacities on DPPH and hydroxyl radicals and the reducing power values. Obviously, both En-MPS and MPS showed concentration-dependent manners on antioxidant effects. As shown in Figures 1(a) and 1(c), the scavenging rates of En-MPS on DPPH and hydroxyl radicals reached $94.35 \pm$ $3.42 \%$ and $70.81 \pm 4.52 \%$, respectively, which were increased by $25.26 \pm 3.69 \%$ and $46.42 \pm 5.65 \%$, respectively, compared to MPS at a concentration of $4,500 \mathrm{mg} / \mathrm{L}$. Meanwhile, EnMPS also showed a stronger reducing power value of 1.24 \pm 0.05 , with $48.32 \pm 0.38 \%$ higher than that of MPS at the concentration of $4,500 \mathrm{mg} / \mathrm{L}$ (Figure $1(\mathrm{~b})$ ). The results indicated that En-MPS showed superior antioxidant effects than MPS.

3.2. In Vivo Antioxidant Activity Assays. The effects of EnMPS and MPS on the activities of SOD, CAT, and T-AOC, as well as the levels of MDA in D-gal-induced aging mice, are presented in Figure 2. Obviously, the activities of SOD, CAT, and T-AOC were significantly decreased, while the levels of MDA were significantly increased in the aging mice (MC groups) when compared with those in the NC groups ( $\mathrm{p}<0.01$ ), illustrating that the aging mice had suffered serious oxidative stress. After a 30-day administration with two polysaccharides, significant elevations $(\mathrm{p}<0.01)$ in SOD, CAT, and T-AOC activities, as well as remarkable descends in MDA, were observed ( $\mathrm{p}<0.01$ ). The SOD, CAT, and TAOC activities in the liver were increased by $129.95 \pm 5.47 \%$, $61.84 \pm 3.56 \%$, and $145.00 \pm 12.96 \%$ after treatment with En-MPS and by $16.59 \pm 3.56 \%, 4.65 \pm 0.96 \%$, and 15.70 $\pm 1.06 \%$ after treatment with MPS at the same dosage of $600 \mathrm{mg} / \mathrm{kg}$, respectively, compared with those in the MC groups. A similar tendency of En-MPS and MPS on SOD, CAT, and T-AOC activities was also observed in the brain and kidney. For mice treated with En-MPS at a high dosage $(600 \mathrm{mg} / \mathrm{kg})$, the inhibition ratios of MDA were decreased by $66.18 \pm 5.65 \%, 45.42 \pm 3.59 \%$, and $33.63 \pm 4.36 \%$ in the brain, liver, and kidney, respectively, showing effective inhibition of lipid peroxidation. All the results showed that En-MPS had superior effects in suppressing oxidative damage in D-galinduced aging mice in a dose-dependent manner than MPS.

3.3. The Serum Biochemistry Assays. The serum levels of TC, TG, HDL-C, and LDL-C were measured to explore the influence of En-MPS and MPS on lipid metabolism, and the results are shown in Figure 3. Visibly, when compared to the NC groups, the levels of TC, TG, and LDL-C significantly 


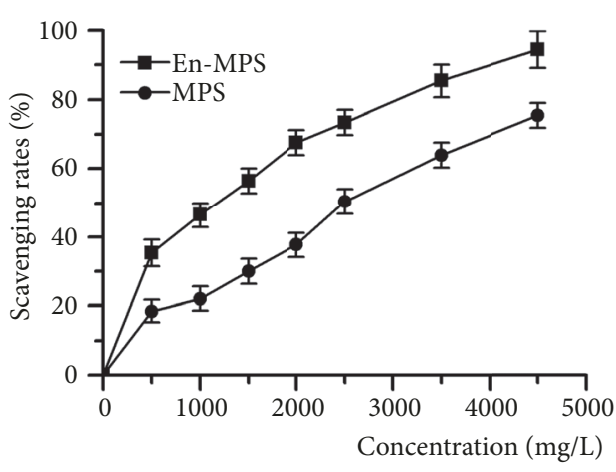

(a)

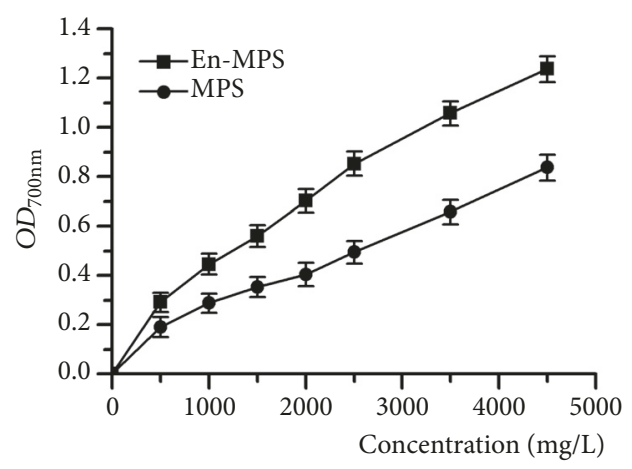

(b)

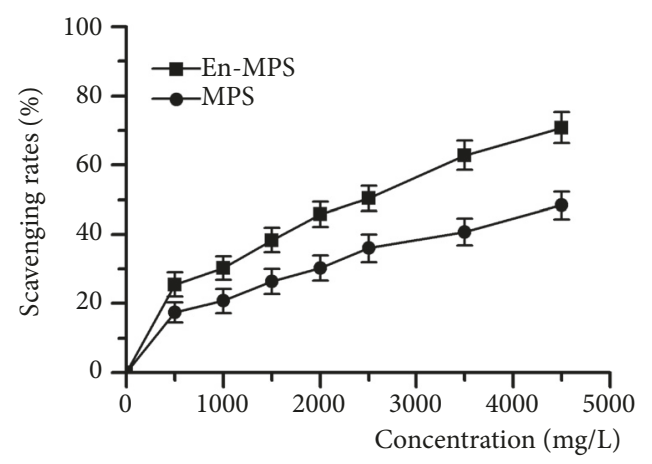

(c)

FIGURE 1: In vitro antioxidant activities of MPS and En-MPS. (a) DPPH radicals, (b) reducing power, and (c) hydroxyl radicals.

increased, while the levels of HDL-C decreased after the Dgal injection $(\mathrm{p}<0.01)$, indicating that the mice suffered severe dysregulation of lipid metabolism. Fortunately, these pathologic changes could be significantly remitted by the administration of En-MPS and MPS ( $p<0.05$ or $\mathrm{p}<0.01$ ). The TC, TG, and LDL-C levels were reduced by $39.28 \pm 5.68 \%$, $43 \pm 2.36 \%$, and $28.57 \pm 4.69 \%$, respectively, while the HDL-C levels were increased by $93.18 \pm 5.58 \%$ when compared with those in the MC groups. When comparing the mice treated with En-MPS at a high dose $(600 \mathrm{mg} / \mathrm{kg})$ with the mice treated with MPS at the same dosage, it was also increased by $4.76 \pm 0.73 \%, 16.67 \pm 3.29 \%$, and $11.4 \pm 2.36 \%$ and decreased by $28.41 \pm 0.78 \%$, respectively. These results indicated that EnMPS showed effects superior to those of MPS in the remission of aging-related lipid metabolism. Furthermore, the ALB levels in aging mice (MC groups) were decreased by 44.78 $\pm 4.08 \%(\mathrm{p}<0.01)$ compared with those in the NC groups. After treatment with the two polysaccharides, the decreased tendency was inhibited, suggesting that both En-MPS and MPS had potential effects on maintaining ALB levels. The enzymatic activities of ALT, AST, and ALP in serum were significantly increased in the MC groups compared to the NC groups $(\mathrm{p}<0.01)$, indicating that the liver had suffered serious damage. As shown in Figure 3(f), the treatment of EnMPS and MPS dose-dependently reduced the D-gal-induced elevation of serum ALT activities, especially En-MPS at a high dose of $600 \mathrm{mg} / \mathrm{kg}$. Meanwhile, the increased AST and ALP activities were also effectively attenuated after En-MPS treatment, which could reach $120.45 \pm 3.32 \mathrm{U} / \mathrm{L}$ and $124.36 \pm$
4.65 U/L at the high dose, respectively. All the data indicated that En-MPS had protective effects against liver damage.

3.4. Effects of En-MPS and MPS on Hyp Contents in Skin. The Hyp contents in skin were investigated by evaluating the effects of polysaccharides on aging skins (Figure 4). After treatment with En-MPS and MPS at the high dose of $600 \mathrm{mg} / \mathrm{kg}$, the Hyp contents significantly increased compared with those in the MC groups $(\mathrm{p}<0.01)$, indicating that the polysaccharides had potent effects on promoting skin collagen synthesis.

3.5. Histopathological Observations. In the present study, histopathological observations of the brain, liver, and kidney were performed to evaluate histological changes (Figures 5 , 6, and 7). Obviously, compared with the normal architectures, including clear staining of the nucleus and cytoplasm (Figures 5(a), 6(a), and 7(a)), the aging mice (MC groups) showed serious organ damage, mainly evidenced by cell damage and nucleus degradation. In addition, the aging mice also showed individual morphologies, such as necrotic cells with loosened and vacuolar neural fibres in the brain (Figure 5(b)), fat vacuole accumulation in the liver (Figure 6(b)), glomerular destruction, and edema of tubular epithelial cells in the kidney (Figure 7(b)). Interestingly, the severe aging-induced damage to the brain, liver, and kidney was considerably prevented at different degrees by the administration of En-MPS and MPS. The results showed that 


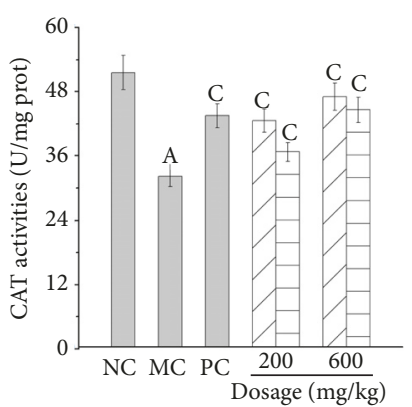

(a)

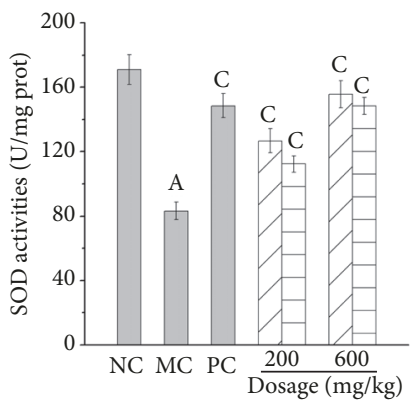

(b)

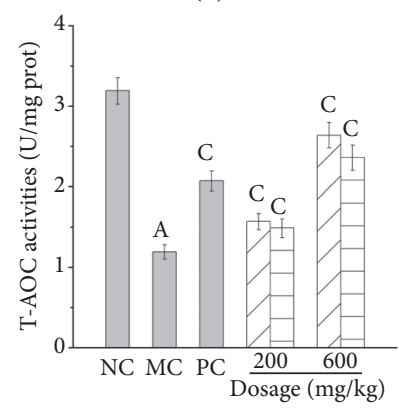

(c)

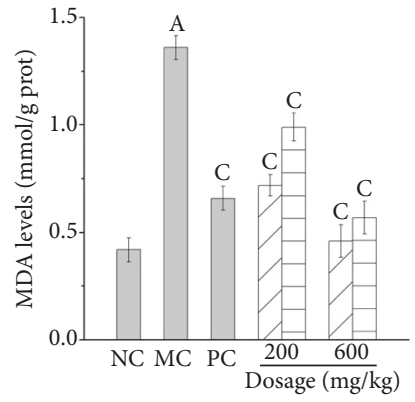

(d)

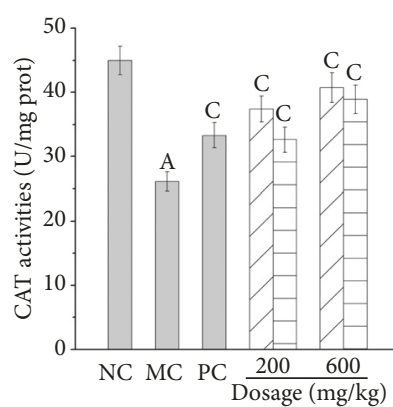

(e)

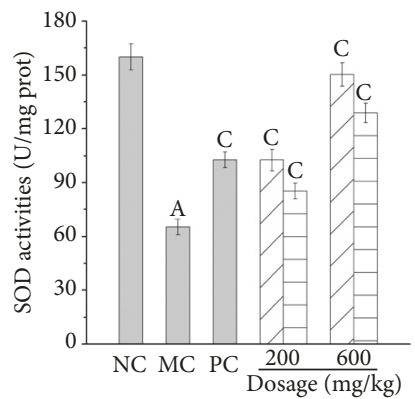

(f)

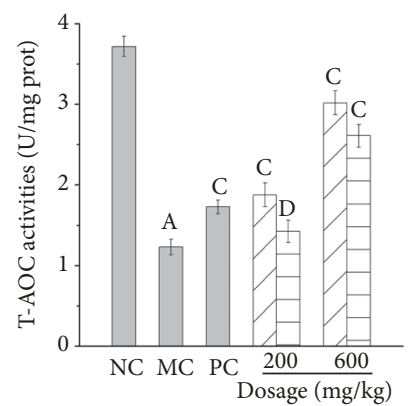

(g)

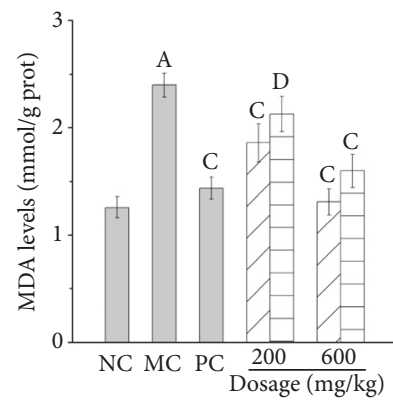

(h)

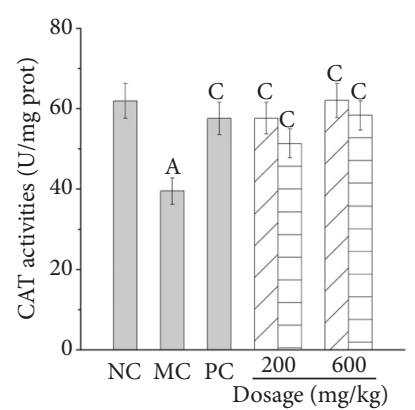

(i)

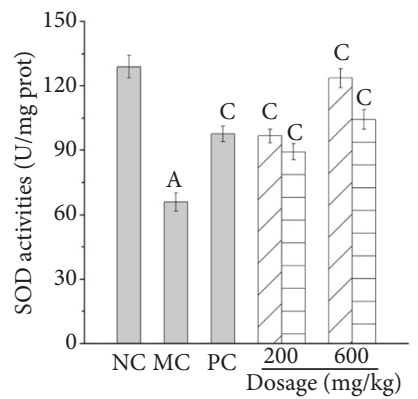

(j)

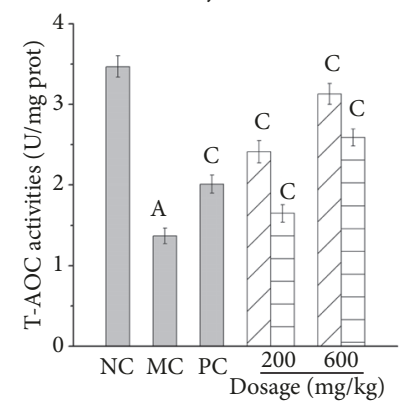

(k)

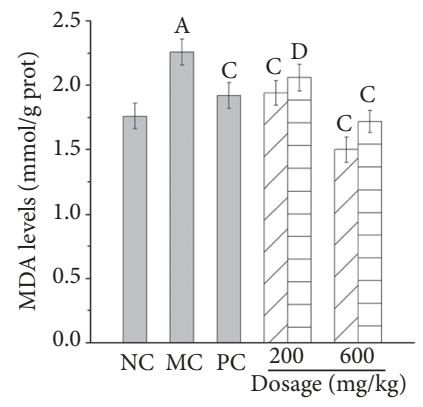

(1)
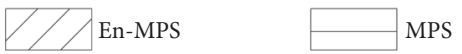

FIGURE 2: The in vivo antioxidant capacities of En-MPS and MPS on brain (a-d), liver (e-h), and kidney (i-l) in D-gal-induced aging mice. The values were reported as the mean \pm SD of five mice per group. (A) $p<0.01$ compared with NC group, (C) p $<0.01$ and (D) $p<0.05$ compared with the MC group.

both En-MPS and MPS can protect these tissues from acute D-gal intoxication.

3.6. Acute Toxicity Study. The mice treated with MPS and En-MPS, even at a dose of $1,500 \mathrm{mg} / \mathrm{kg}$, did not exhibit any significant changes in behavioural, autonomic, or toxic responses. Furthermore, no deaths were observed at the end of the experiment or during the experimental period. These results indicated that both MPS and En-MPS were practically nontoxic substances. 


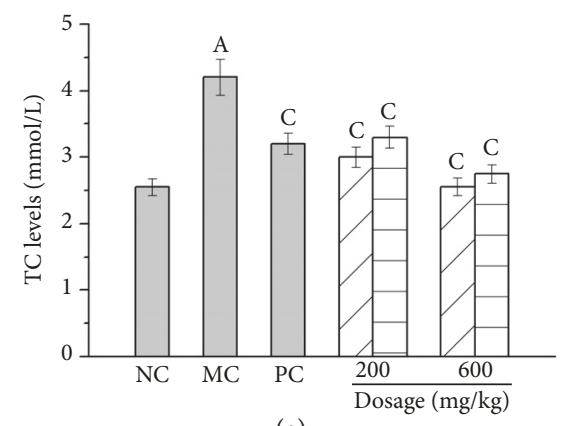

(a)

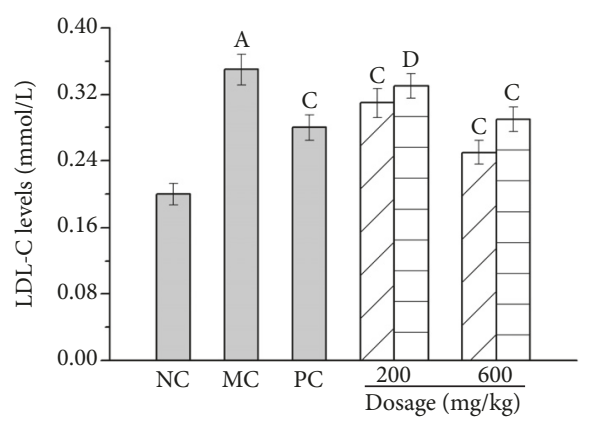

(c)

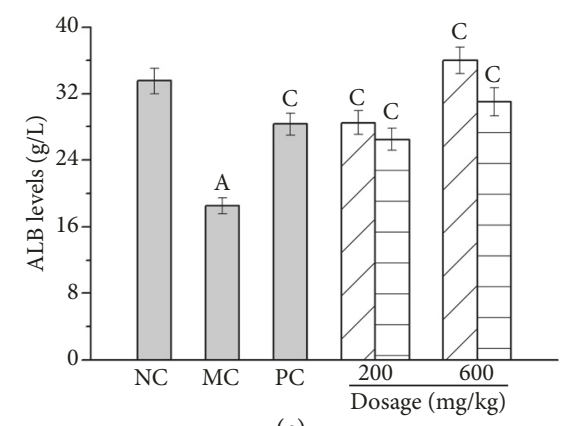

(e)

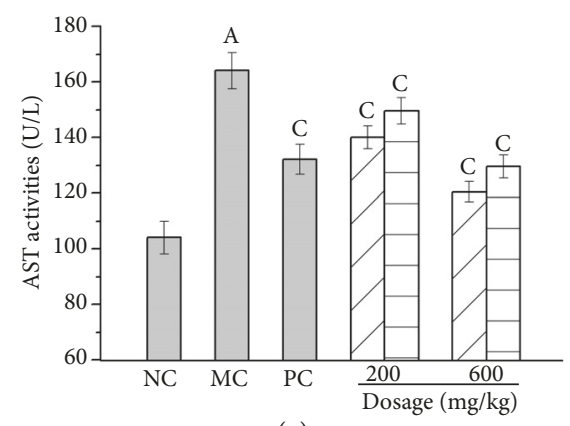

(g)

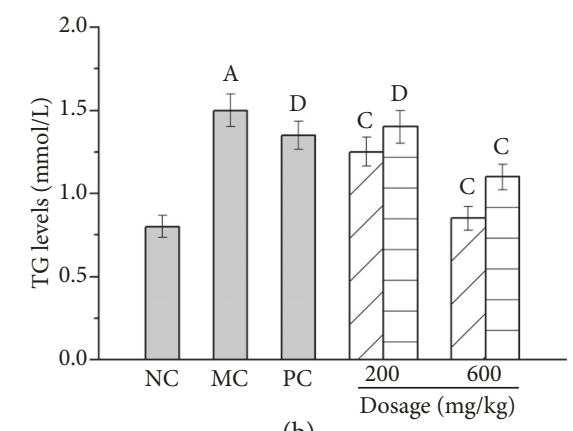

(b)

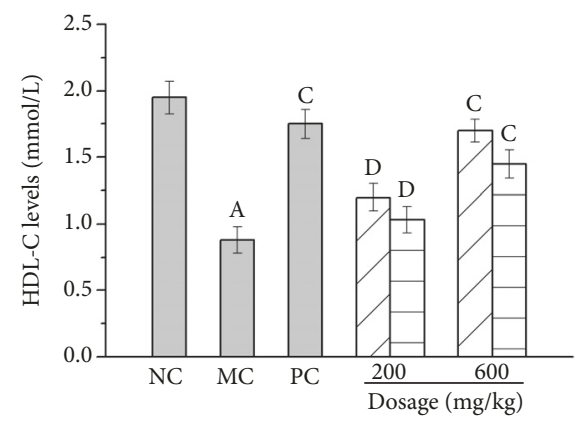

(d)

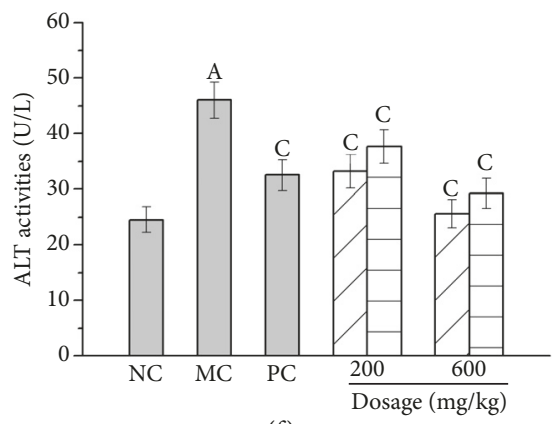

(f)

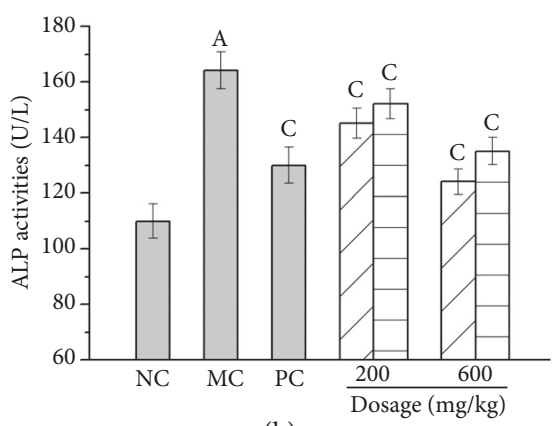

(h) MPS

FIGURE 3: Effects of En-MPS and MPS on serum levels of TC (a), TG (b), LDL-C (c), HDL-C (d), and ALB (e), and activities of ALT (f), AST $(\mathrm{g})$, and ALP $(\mathrm{h})$ in D-gal-induced aging mice. The values were reported as the mean \pm SD of five mice per group. (A) $\mathrm{p}<0.01 \mathrm{compared}$ with NC group, (C) p $<0.01$ and (D) $\mathrm{p}<0.05$ compared with the MC group.

3.7. Chemical Composition. The chemical composition of MPS and En-MPS, including the contents of total polysaccharides, protein, and total phenolic, was analyzed, and the results were listed in Table 1. As shown in Table 1, polysaccharides were the main compounds of MPS and
En-MPS, and the contents were $83.11 \pm 3.85 \%$ and $89.82 \pm$ $4.12 \%$, respectively. Moreover, MPS contained $2.09 \pm 0.41 \%$ protein and $0.21 \pm 0.02 \%$ total phenolic, while En-MPS contained $4.01 \pm 0.34 \%$ protein and $0.36 \pm 0.08 \%$ total phenolic. The results above indicated that the extraction 


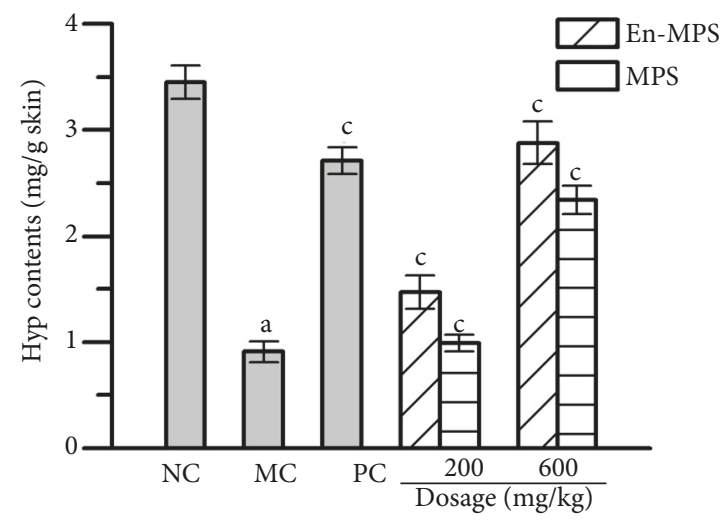

Figure 4: Effects of En-MPS and MPS on the Hyp contents in the D-gal-induced aging skins. The values were reported as the mean \pm SD of five mice per group. (a) $\mathrm{p}<0.01$ compared with $\mathrm{NC}$ group, and (c) $\mathrm{p}<0.01$ compared with the MC group.

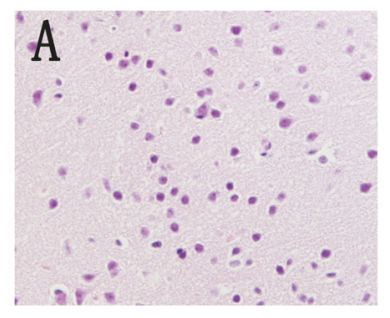

(a)

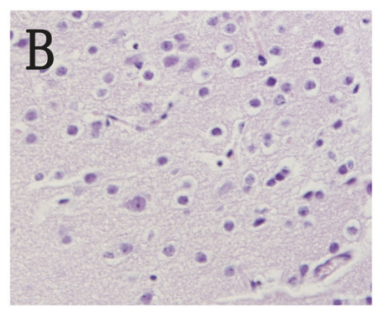

(b)

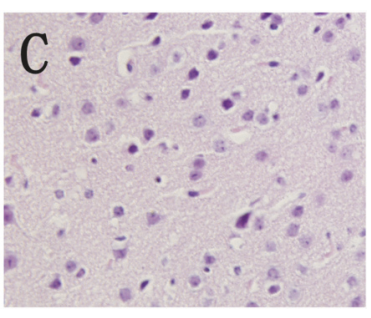

(c)

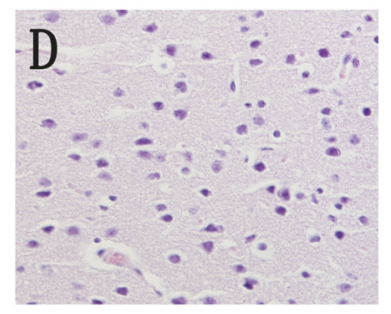

(d)

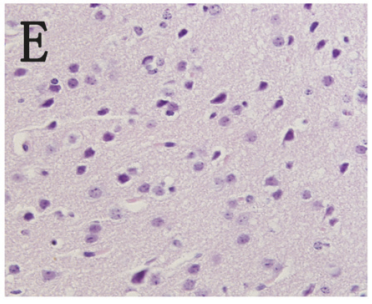

(e)

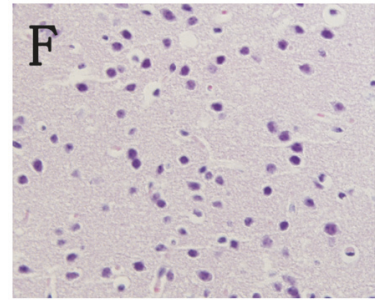

(f)

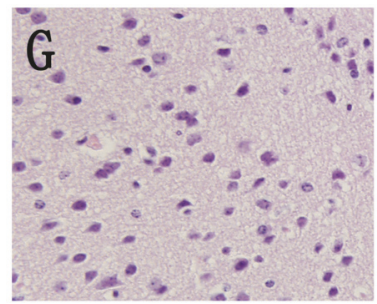

(g)

FIGURE 5: Morphological changes of brain in D-gal-induced aging mice. NC groups (a), MC groups (b), PC group (c), MPS at $200 \mathrm{mg} / \mathrm{kg}$ (d), MPS at $600 \mathrm{mg} / \mathrm{kg}(\mathrm{e}), \mathrm{En}-\mathrm{MPS}$ at $200 \mathrm{mg} / \mathrm{kg}$ (f), and En-MPS at $600 \mathrm{mg} / \mathrm{kg}$ (g) with original magnification of $400 \mathrm{X}$.

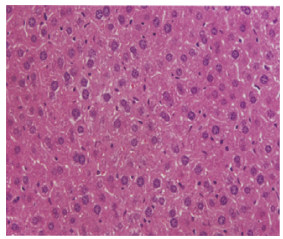

(a)

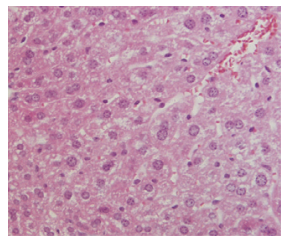

(b)

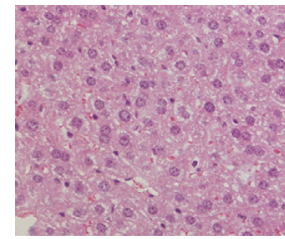

(c)

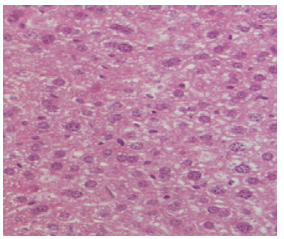

(d)

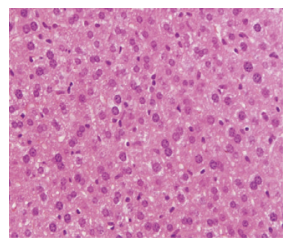

(e)

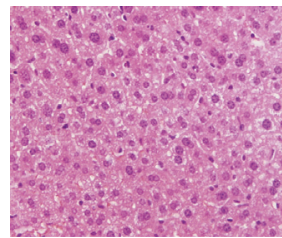

(f)

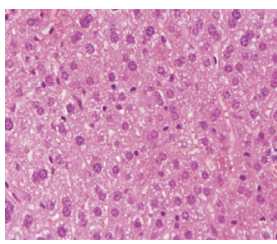

(g)

FIGURE 6: Morphological changes of liver in D-gal-induced aging mice. NC groups (a), MC groups (b), PC groups (c), MPS at $200 \mathrm{mg} / \mathrm{kg}$ (d), MPS at $600 \mathrm{mg} / \mathrm{kg}(\mathrm{e})$, En-MPS at $200 \mathrm{mg} / \mathrm{kg}$ (f), and En-MPS at $600 \mathrm{mg} / \mathrm{kg}$ (g) with original magnification of 400X. 


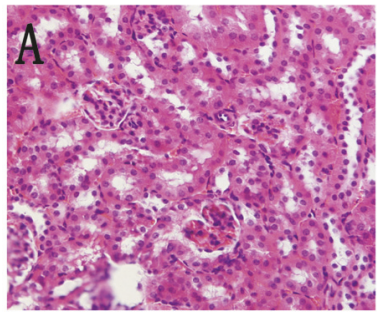

(a)

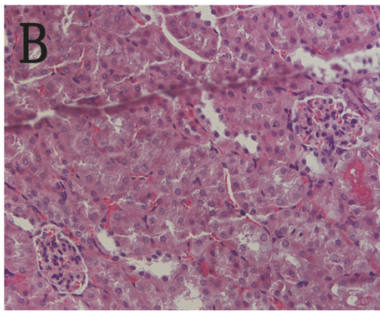

(b)

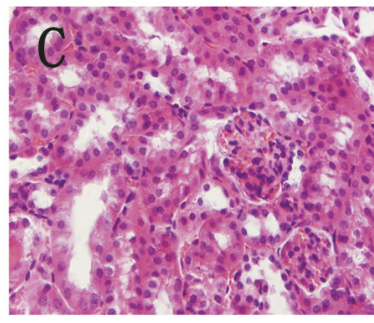

(c)

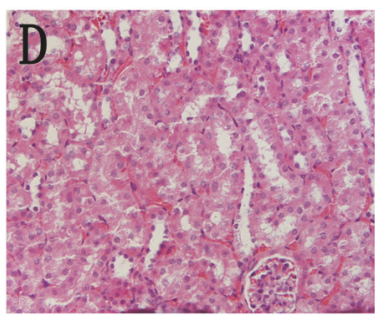

(d)

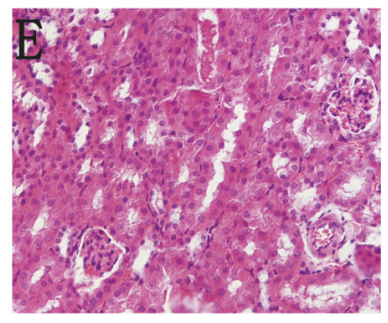

(e)

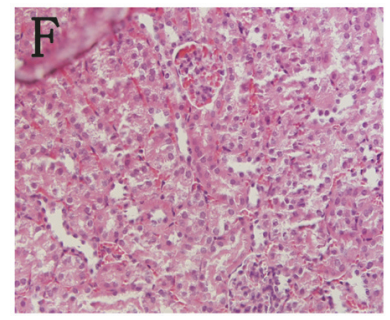

(f)

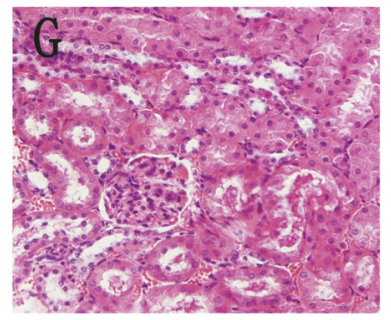

(g)

FIGURE 7: Morphological changes of kidney in D-gal-induced aging mice. NC groups (a), MC groups (b), PC groups (c), MPS at 200 mg/kg (d), MPS at $600 \mathrm{mg} / \mathrm{kg}$ (e), En-MPS at $200 \mathrm{mg} / \mathrm{kg}$ (f), and En-MPS at $600 \mathrm{mg} / \mathrm{kg}$ (g) with original magnification of $400 \mathrm{X}$.

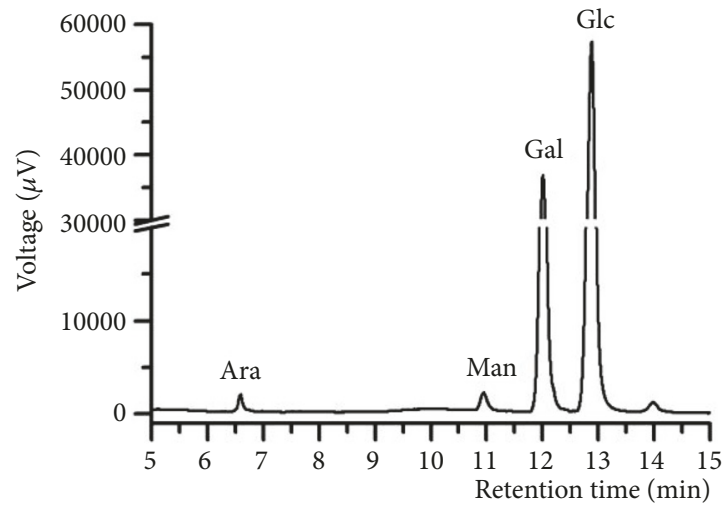

(a)

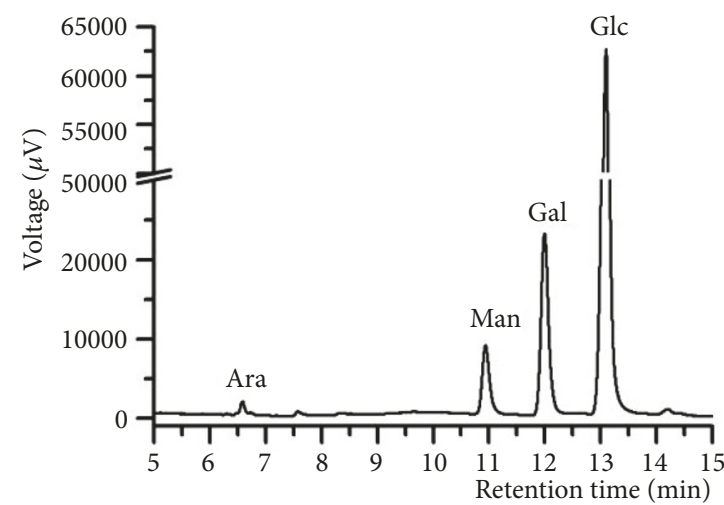

(b)

FIGURE 8: GC chromatograms of MPS (a), En-MPS (b).

technology had an effect on chemical composition of MPS and En-MPS.

3.8. Monosaccharide Composition Analysis. The GC chromatograms of MPS and En-MPS are shown in Figure 8. Obviously, both MPS and En-MPS were composed of Ara, Man, Gal, and Glc with molar ratios of 1:1.5:17:58.8 and 1:8.2:24.3:77, respectively. The results showed that the major component was Glc in both MPS and En-MPS, and the contents of monosaccharides in En-MPS were more abundant than in MPS, indicating that the monosaccharide compositions were involved in maintaining the antioxidant effects.

\section{Discussion}

With the improvement of living standards, people have paid more attention to their appearance and health. Hence, how to successfully delay aging has become a hot topic. In the present study, D-gal was used to induce an aging model owing to its toxicity in inducing the generation of free radicals in vivo, which could break the intrinsic balance, resulting in aging [3]. A huge amount of evidence has indicated that aging is associated with decreases in antioxidant activities and increases in lipid peroxidation, which are all caused by metabolic disequilibrium of free radicals such as DPPH and hydroxyl radicals $[3,30]$. A previous study has argued that reducing power is also an indicator of antioxidants by breaking the free radical chains [31]. Thus, the scavenging capacities on these radicals seem to be important for the evaluation of natural substances. In the present study, En-MPS exhibited stronger antioxidant capacities than MPS, reflecting higher free radical-scavenging abilities and reducing power values

Meanwhile, as the major antioxidant enzymes, SOD and CAT have been shown to be involved in the first defence 
TABLE 1: The polysaccharides, protein, and phenolic contents of MPS and En-MPS.

\begin{tabular}{lccc}
\hline Samples & Total polysaccharide (\%) & Protein (\%) & Total phenolic (\%) \\
\hline MPS & $83.11 \pm 3.85$ & $2.90 \pm 0.41$ & $0.21 \pm 0.02$ \\
\hline En-MPS & $89.82 \pm 4.12$ & $4.01 \pm 0.34$ & $0.36 \pm 0.08$ \\
\hline
\end{tabular}

line in eliminating ROS-induced oxidative stress [32]. Both SOD and CAT play vital roles in protecting cells from ROS-mediated damage, contributing to the functions of removing superoxide anions and catalysing hydrogen peroxide into water and oxygen, respectively [14]. Additionally, the nonenzymatic antioxidant systems (T-AOC) also play vital roles in maintaining the antioxidant status [33]. As a primary parameter of oxidative damage, MDA is regarded as a sensitive biomarker because lipid peroxidation could lead to hydroperoxide generation [34]. In the present study, the activities of CAT, SOD, and T-AOC were significantly increased, while the contents of MDA were remarkably decreased, suggesting that both En-MPS and MPS could remarkably protect the mice against oxidative stress and that En-MPS showed superior effects.

Clinically, previous studies have shown that higher serum lipids, including TC, TG, and LDL-C, are believed to be related to cardiovascular diseases, coronary heart diseases, and blood viscosity $[35,36]$. Lower HDL-C levels could retard the metabolism of cholesterol, accelerating the accumulation of serum lipids and the development of atherosclerosis [37]. Thus, decreased HDL-C levels, as well as increased LDL-C, TC, and TG levels in serum, are deemed significant clinical signs of hyperlipidaemia, atherosclerosis, and cardiovascular diseases [36]. It was found that in the present study, both EnMPS and MPS had potential effects in significantly decreasing TC, TG, and LDL-C levels and increasing HDL-C levels, indicating that the polysaccharides showed potential advantages in the treatment of aging-related diseases. Furthermore, low serum ALB levels have been regarded as a marker of diminishing protein severity and loss of skeletal muscle mass [6]. Therefore, it is vital to increase serum ALB concentrations. In the present study, the decrease in ALB concentrations in D-gal-induced aging mice was significantly elevated by polysaccharide administration $(\mathrm{p}<0.01)$, suggesting that EnMPS showed superior effects on maintaining serum ALB levels. The activities of AST, ALT, and ALP in serum are commonly used as biochemical markers for early acute hepatic damage, and it was markedly heightened when liver damage occurred $[18,38]$. Currently, the significant increases in ALT, AST, and ALP activities were reduced after treatment with En-MPS and MPS, indicating that polysaccharides from A. aegerita (Brig.) Sing had potential activities in preventing liver from aging-related damage.

As the largest protective barrier of the human body, the skin is sensitive to aging, and collagen and skin elasticity decline when aging occurs $[3,39]$. Experimentally, Hyp is a major component of collagen that plays a key role in collagen stability [40]. In the current study, a significant increase in Hyp contents was observed when the mice were administered En-MPS, suggesting that En-MPS could improve the metabolism of skin against aging toxicity.
Polysaccharides are considered as effective antioxidants with a complex structure. In some reports, natural polysaccharides always conjugate with other bioactive constituents, such as protein, phenolic, amino acid, and lipids [41]. Researches have confirmed that there is a positive correlation between antioxidant capacities and the content of phenolic or protein compounds conjugated in the polysaccharide extracts of macro fungi $[42,43]$. The present study showed that the contents of total polysaccharides, protein, and total phenolic in En-MPS were higher than those in MPS, and the composition of En-MPS components was near 90\%, while MPS included unidentified components. En-MPS with higher purity showed a stronger antioxidant capacity than MPS. Previous study had demonstrated that polysaccharides with high purity showed strong antioxidant activities [44]. Therefore we could presume that the antioxidant activities of En-MPS and MPS showed a significant correlation to their purity.

Also, the antioxidant capacities of polysaccharides could be influenced by extraction solvent [43]. In some reports, antioxidant capacities of polysaccharides extracted with snailase solution (4\%) were higher than polysaccharides extracted with other solutions [19, 45]. In this study, we found that polysaccharides extracted with snailase solution (4\%) showed a higher antioxidant capacities, which was in agreement with the result of $\mathrm{Xu}$ et al. [18]. Compared with our previous study [29], the antioxidant capacities of EnMPS were higher than the polysaccharides extracted with acid and alkalic solutions, which may be due to different chemical compositions of polysaccharides caused by different extraction technology.

In addition, it has been demonstrated that the antioxidant effects of polysaccharides could be influenced by the monosaccharide compositions [46]. In the present study, the results showed that both MPS and En-MPS were composed of Ara, Man, Gal, and Glc. En-MPS with high purity contained higher contents of Man, Gal, and Glc, and En-MPS showed a stronger antioxidant capacity than MPS. Similar results were also reported by Xu et al. [18], in which enzymatic-extractable mycelia zinc polysaccharides (En-MZPS) of Pleurotus eryngii var. tuoliensis with higher Man, Gal, and Glc contents showed a stronger antioxidant capacity than enzymatic-extractable mycelia polysaccharides (En-MPS) of Pleurotus eryngii var. tuoliensis.

\section{Conclusion}

In conclusion, both En-MPS and MPS by A. aegerita (Brig.) Sing showed potential antioxidant, antiaging, and organic protective effects on the brain, liver, and kidney against D-galinduced aging toxicities, and differences in biological activity of MPS and En-MPS showed a significant correlation to their 
purity. En-MPS and MPS could be exploited as natural and functional foods for the prevention and alleviation of aging and its complications. In addition, these findings also provide an available reference for the mechanisms of antioxidation and antiaging.

\begin{tabular}{ll} 
Abbreviations \\
ALT: & Alanine aminotransferase \\
ALB: & Albumin \\
ALP: & Alkaline phosphatase \\
Ara: & Arabinose \\
AST: & Aspartate aminotransferase \\
CAT: & Catalase \\
D-gal: & D-galactose \\
En-MPS: & Enzymatic-extractable mycelium \\
& polysaccharides \\
Fuc: & Fucose \\
Gal: & Galactose \\
GC: & Gas chromatography \\
Glc: & Glucose \\
HDL-C: & High-density lipoprotein cholesterol \\
Hyp: & Hydroxyproline \\
LDL-C: & Low-density lipoprotein cholesterol \\
MDA: & Malondialdehyde \\
Man: & Mannose \\
MC group: & Model control group \\
MPS: & Mycelium polysaccharides \\
NC group: & Normal control group \\
PC group: & Positive control group \\
ROS: & Reactive oxygen species \\
Rha: & Rhamnose \\
Rib: & Ribose \\
SOD: & Superoxide dismutase \\
T-AOC: & Total antioxidant capacity \\
TC: & Total cholesterol \\
TG: & Triacylglycerol \\
Xyl: & Xylose. \\
& \\
\hline &
\end{tabular}

\section{Data Availability}

The data used to support the findings of this study are available from the corresponding author upon request.

\section{Conflicts of Interest}

The authors declared no conflicts of interest.

\section{Authors' Contributions}

Huijuan Jing, Qing Zhang, and Min Liu are equal contributors.

\section{Acknowledgments}

This work was supported by grants from Mushroom Technology System of Shandong Province (SDAIT-07-05).

\section{References}

[1] D. Harman, "Aging: a theory based on free radical and radiation chemistry," Journal of Gerontology, vol. 11, no. 3, pp. 298-300, 1956.

[2] C. Alvarado, P. Álvarez, L. Jiménez, and M. De la Fuente, "Oxidative stress in leukocytes from young prematurely aging mice is reversed by supplementation with biscuits rich in antioxidants," Developmental \& Comparative Immunology, vol. 30, no. 12, pp. 1168-1180, 2006.

[3] Y. Ye, R.-R. Jia, L. Tang, and F. Chen, "In vivo antioxidant and anti-skin-aging activities of ethyl acetate extraction from idesia polycarpa defatted fruit residue in aging mice induced by D-galactose," Evidence-Based Complementary and Alternative Medicine, vol. 2014, 2014.

[4] W.-S. Lin, J.-Y. Chen, J.-C. Wang et al., “The anti-aging effects of Ludwigia octovalvis on Drosophila melanogaster and SAMP8 mice," AGE, vol. 36, no. 2, pp. 689-703, 2014.

[5] J. Fan, H. Feng, Y. Yu et al., "Antioxidant activities of the polysaccharides of Chuanminshen violaceum," Carbohydrate Polymers, vol. 157, pp. 629-636, 2017.

[6] M. Visser, S. B. Kritchevsky, A. B. Newman et al., "Lower serum albumin concentration and change in muscle mass: The Health, Aging and Body Composition Study," American Journal of Clinical Nutrition, vol. 82, no. 3, pp. 531-537, 2005.

[7] F. B. Johnson, D. A. Sinclair, and L. Guarente, "Molecular biology of aging," Cell, vol. 96, no. 2, pp. 291-302, 1999.

[8] W. Zhong, N. Liu, Y. Xie, Y. Zhao, X. Song, and W. Zhong, "Antioxidant and anti-aging activities of mycelial polysaccharides from Lepista sordida," International Journal of Biological Macromolecules, vol. 60, pp. 355-359, 2013.

[9] Z. Yan-Chun and Z. Rong-Liang, "Phenolic compounds and an analog as superoxide anion scavengers and anti oxidants," Biochemical Pharmacology, vol. 42, no. 6, pp. 1177-1179, 1991.

[10] M. Friedman, "Mushroom Polysaccharides: Chemistry and Antiobesity, Antidiabetes, Anticancer, and Antibiotic Properties in Cells, Rodents, and Humans," Foods, vol. 5, no. 4, p. 80, 2016.

[11] C. Chang, C. Lin, and C. Lu, "Ganoderma lucidum reduces obesity in mice by modulating the composition of the gut microbiota," Nature Communications, vol. 23, article 7489, no. 6, 2015.

[12] A. T. M. Lima, M. N. Santos, L. A. R. De Souza et al., "Chemical characteristics of a heteropolysaccharide from Tylopilus ballouii mushroom and its antioxidant and anti-inflammatory activities," Carbohydrate Polymers, vol. 144, pp. 400-409, 2016.

[13] N. Xu, Z. Ren, J. Zhang et al., "Antioxidant and antihyperlipidemic effects of mycelia zinc polysaccharides by Pleurotus eryngii var. tuoliensis," International Journal of Biological Macromolecules, vol. 95, pp. 204-214, 2017.

[14] Q. Ding, D. Yang, W. Zhang et al., "Antioxidant and antiaging activities of the polysaccharide TLH-3 from Tricholoma lobayense," International Journal of Biological Macromolecules, vol. 85, pp. 133-140, 2016.

[15] D. S. Li, P. Li, Z. Wang, and J. Su, "Physicochemical properties of antimicrobial substances from Agrocybe aegerita," Journal of Shandong University, vol. 42, p. 10, 2017.

[16] J. Petrović, J. Glamočlija, D. Stojković et al., "Bioactive composition, antimicrobial activities and the influence of Agrocybe aegerita (Brig.) Sing on certain quorum-sensing-regulated functions and biofilm formation by Pseudomonas aeruginosa," Food \& Function, vol. 5, no. 12, pp. 3296-3303, 2014. 
[17] L. Shi, "Bioactivities, isolation and purification methods of polysaccharides from natural products: A review," International Journal of Biological Macromolecules, vol. 92, pp. 37-48, 2016.

[18] N. Xu, Z. Gao, J. J. Zhang et al., "Hepatoprotection of enzymaticextractable mycelia zinc polysaccharides by Pleurotus eryngii var. tuoliensis," Carbohydrate Polymers, vol. 157, pp. 196-206, 2017.

[19] L. Lin, F. Cui, J. Zhang et al., "Antioxidative and renoprotective effects of residue polysaccharides from Flammulina velutipes," Carbohydrate Polymers, vol. 146, pp. 388-395, 2016.

[20] M. Dubois, K. A. Gilles, J. K. Hamilton, P. A. Rebers, and F. Smith, "Colorimetric method for determination of sugars and related substances," Analytical Chemistry, vol. 28, no. 3, pp. 350$356,1956$.

[21] A. M. Staub, "Removal of proteins: sevag method," Methods in Carbohydrate Chemistry, vol. 5, p. 5, 1965.

[22] L. Zheng, M. Liu, G.-Y. Zhai, Z. Ma, L.-Q. Wang, and L. Jia, "Antioxidant and anti-ageing activities of mycelia zinc polysaccharide from Pholiota nameko SW-03," Journal of the Science of Food and Agriculture, vol. 95, no. 15, pp. 3117-3126, 2015.

[23] M. Oyaizu, "Studies on products of browning reaction: antioxidative activity of products of browning reaction prepared from glucosamine," The Japanese Journal of Nutrition and Dietetics, vol. 44, pp. 307-315, 1986.

[24] N. Smirnoff and Q. J. Cumbes, "Hydroxyl radical scavenging activity of compatible solutes," Phytochemistry, vol. 28, no. 4, pp. 1057-1060, 1989.

[25] J. Chao, T.-C. Lu, J.-W. Liao et al., "Analgesic and antiinflammatory activities of ethanol root extract of Mahonia oiwakensis in mice," Journal of Ethnopharmacology, vol. 125, no. 2, pp. 297-303, 2009.

[26] M. M. Bradford, "Rapid and sensitive method for the quantitation of microgram quantities of protein utilizing the principle of protein-dye binding," Analytical Biochemistry, vol. 72, no. 1-2, pp. 248-254, 1976.

[27] V. L. Singleton and J. A. Rossi, "Colorimetry of total phenolics with phosphomolybdic-phosphotungstic acid reagents," American Journal of Enology and Viticulture, vol. 16, pp. 144-158, 1965.

[28] J. Sheng, F. Yu, Z. Xin, L. Zhao, X. Zhu, and Q. Hu, "Preparation, identification and their antitumor activities in vitro of polysaccharides from Chlorella pyrenoidosa," Food Chemistry, vol. 105, no. 2, pp. 533-539, 2007.

[29] H. Jing, J. Li, J. Zhang et al., "The antioxidative and anti-aging effects of acidic- and alkalic-extractable mycelium polysaccharides by Agrocybe aegerita (Brig.) Sing," International Journal of Biological Macromolecules, vol. 106, pp. 1270-1278, 2018.

[30] C. Zhang, Z. Gao, C. Hu et al., "Antioxidant, antibacterial and anti-aging activities of intracellular zinc polysaccharides from Grifola frondosa SH-05," International Journal of Biological Macromolecules, vol. 95, pp. 778-787, 2017.

[31] S. Meir, J. Kanner, B. Akiri, and S. Philosoph-Hadas, "Determination and involvement of aqueous reducing compounds in oxidative defense systems of various senescing leaves," Journal of Agricultural and Food Chemistry, vol. 43, no. 7, pp. 1813-1819, 1995.

[32] S. Govindan, E. E. R. Johnson, J. Christopher, J. Shanmugam, V. Thirumalairaj, and J. Gopalan, "Antioxidant and anti-aging activities of polysaccharides from Calocybe indica var. APK2," Experimental and Toxicologic Pathology, vol. 68, no. 6, pp. 329334, 2016.
[33] Y. Tian, B. Zou, L. Yang et al., "High molecular weight persimmon tannin ameliorates cognition deficits and attenuates oxidative damage in senescent mice induced by D-galactose," Food and Chemical Toxicology, vol. 49, no. 8, pp. 1728-1736, 2011.

[34] J. Zhang, M. Liu, Y. Yang et al., "Purification, characterization and hepatoprotective activities of mycelia zinc polysaccharides by Pleurotus djamor," Carbohydrate Polymers, vol. 136, Article ID 10377, pp. 588-597, 2016.

[35] A. Kitamura, H. Iso, Y. Naito et al., "High-density lipoprotein cholesterol and premature coronary heart disease in urban Japanese men," Circulation, vol. 89, no. 6, pp. 2533-2539, 1994.

[36] X. Liu, Z. Sun, M. Zhang et al., "Antioxidant and antihyperlipidemic activities of polysaccharides from sea cucumber Apostichopus japonicus," Carbohydrate Polymers, vol. 90, no. 4, pp. 1664-1670, 2012.

[37] H. Zhao, S. Li, J. Zhang et al., “The antihyperlipidemic activities of enzymatic and acidic intracellular polysaccharides by Termitomyces albuminosus," Carbohydrate Polymers, vol. 151, pp. 12271234, 2016.

[38] C. Jiang, Q. Xiong, D. Gan et al., "Antioxidant activity and potential hepatoprotective effect of polysaccharides from Cyclina sinensis," Carbohydrate Polymers, vol. 91, no. 1, pp. 262268, 2013.

[39] Longyuan $\mathrm{Hu}$, Jia Tan, Xiaomei Yang et al., "Polysaccharide Extracted from Laminaria japonica Delays Intrinsic Skin Aging in Mice," Evidence-Based Complementary and Alternative Medicine, vol. 2016, Article ID 5137386, 8 pages, 2016.

[40] W. Hu, D. Dai, and W. Li, "Anti-aging effect of Blakeslea trispora powder on adult mice," Biotechnology Letters, vol. 35, no. 8, pp. 1309-1315, 2013.

[41] J. Q. Wang, S. Z. Hu, S. P. Nie, Q. Yu, and M. Y. Xie, "Reviews on mechanisms of in vitro antioxidant activity of polysaccharides," Oxidative Medicine \& Cellular Longevity, vol. 2016, Article ID 5692852, 2016.

[42] K. C. Siu, X. Chen, and J. Y. Wu, "Constituents actually responsible for the antioxidant activities of crude polysaccharides isolated from mushrooms," Journal of Functional Foods, vol. 11, pp. 548-556, 2014.

[43] S. K. Bøhn, N. C. Ward, J. M. Hodgson, and K. D. Croft, "Effects of tea and coffee on cardiovascular disease risk," Food \& Function, vol. 3, no. 6, pp. 575-591, 2012.

[44] Y. Chen, M.-Y. Xie, S.-P. Nie, C. Li, and Y.-X. Wang, "Purification, composition analysis and antioxidant activity of a polysaccharide from the fruiting bodies of Ganoderma atrum," Food Chemistry, vol. 107, no. 1, pp. 231-241, 2008.

[45] J. Zhang, G. Meng, C. Zhang et al., "The antioxidative effects of acidic-, alkalic-, and enzymatic-extractable mycelium zinc polysaccharides by Pleurotus djamor on liver and kidney of streptozocin-induced diabetic mice," BMC Complementary and Alternative Medicine, vol. 15, no. 1, 2015.

[46] C. M. P. Guerra Dore, M. G. D. C. F. Alves, M. D. G. L. Santos, L. A. R. De Souza, I. G. Baseia, and E. L. Leite, "Antioxidant and anti-inflammatory properties of an extract rich in polysaccharides of the mushroom Polyporus dermoporus," Antioxidants, vol. 3, no. 4, pp. 730-744, 2014. 


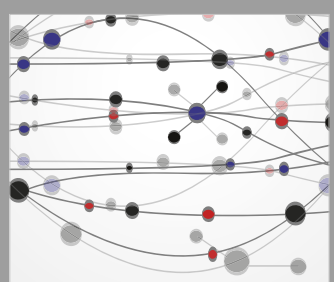

The Scientific World Journal
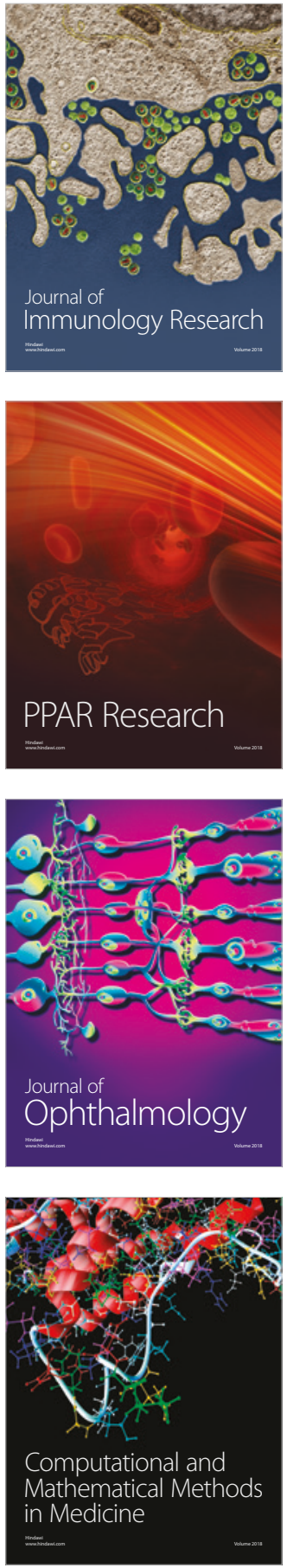

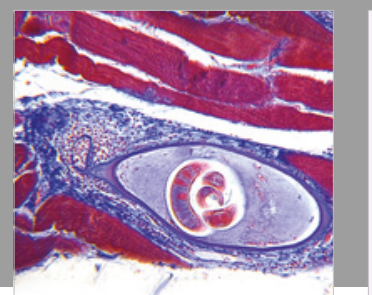

Gastroenterology Research and Practice

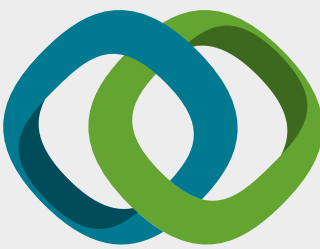

\section{Hindawi}

Submit your manuscripts at

www.hindawi.com
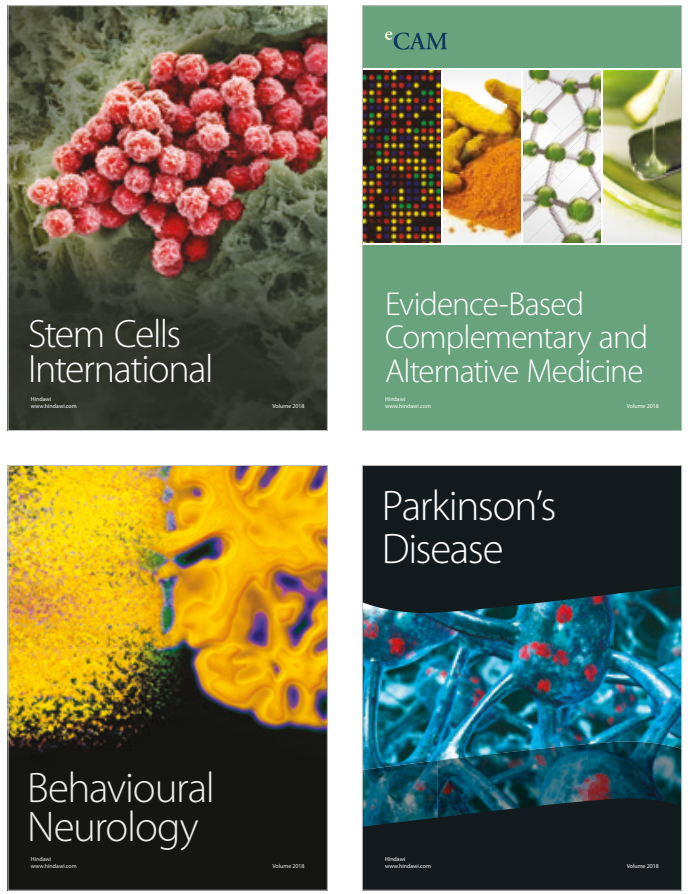

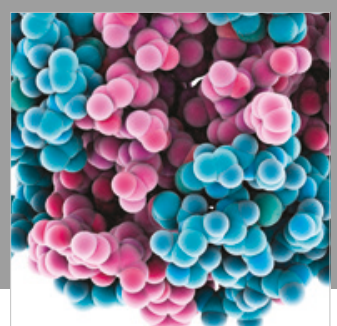

ournal of

Diabetes Research

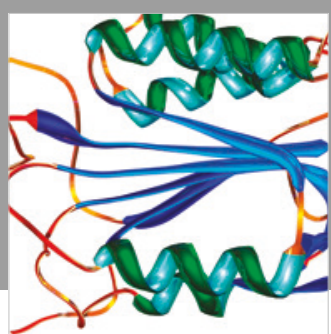

Disease Markers
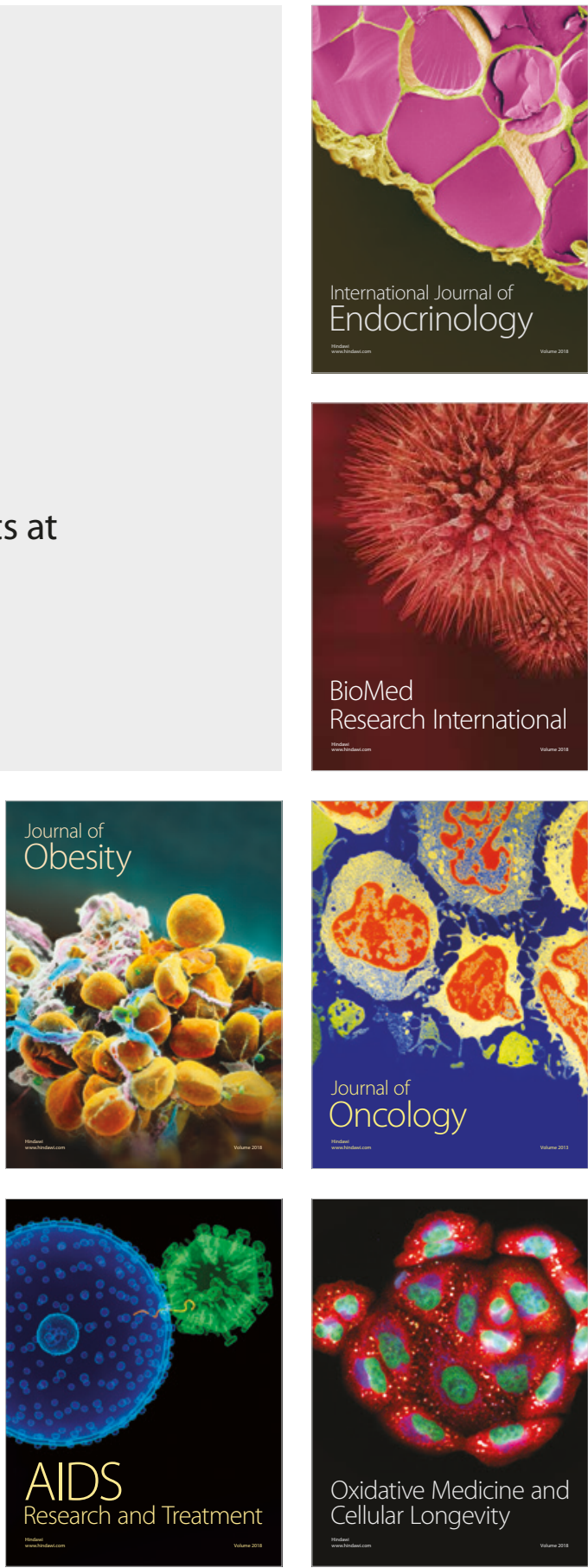